\title{
LABORATORY OBSERVATIONS OF TRIAD INTERACTION OF DEEP WATER WIND WAVES
}

\author{
KYUNG DOUG SUH, SANG HO OH \\ School of Civil, Urban, and Geosystem Engineering, Seoul National University, \\ Seoul 151-742, Korea \\ NORIAKI HASHIMOTO \\ Hydrodynamics Laboratory, Port and Harbour Research Institute, Ministry of Transport, 3-1-1, \\ Nagase, Yokosuka, 239-0826 Japan \\ KYUNGMO AHN \\ School of Construction, Urban and Environmental Engineering, Handong University, \\ Pohang, Kyungbuk 791-940, Korea
}




\begin{abstract}
The triad interactions have been known to be important only for shoaling waves or finite depth wind waves. In deep water, they are insignificant compared with the quadruplet interactions in respect to the evolution of wind waves due to energy transfer among the wave components. However, the triad interactions may be important even for deep water waves because they may closely be related to the wave steepness, which definitely affects wave breaking, drag of air flow over the sea, or navigation of ships, especially during the early stage of the development of wind waves. This study reports a series of laboratory experiments, whose data are subjected to bispectral analyses to investigate the triad interactions of deep-water wind waves. It is found that the bicoherence at the spectral peak frequency and the wave steepness are almost directly proportional, indicating that the steep waves with peaked crests and flat troughs are resulted from the triad interactions. Both bicoherence and wave steepness increase with the wave age during the early stage of wave generation and then drop off as the waves grow old. It seems that the energy of the secondary spectral peak developed by the triad interactions during the early stage of wave generation is redistributed to the neighboring frequencies by the quadruplet interactions during the later stage.
\end{abstract}

Keywords: Bispectra, laboratory tests, phase coherence, wave interactions, wave steepness, wind waves. 


\section{Introduction}

The importance of triad (three wave) interactions for shoaling waves has been reported by several investigators (Elgar and Guza, 1985; Freilich et al., 1990; Ahn, 1993; Elgar et al., 1995). They observed the appearance of harmonics of the spectral peak of shoaling waves, which are phase coherent with the spectral peak, indicating they are the result of triad interactions. Recently, on the other hand, Young and Eldeberky (1998) showed that the triad interactions are also significant for finite depth wind waves generated on essentially constant depth water.

For deep water waves, Phillips (1960) analytically showed that the secondary waves due to triad interactions are always bounded in time and their amplitudes are small compared with those of primary waves, while the tertiary waves due to quadruplet (four wave) interactions can be linearly grown with time in a resonant manner so that their amplitudes can be as large as those of primary waves. Therefore, although the intensity of quadruplet interactions is one order of magnitude smaller than that of triad interactions, their cumulative dynamical effect is much more profound because of their resonant behavior. Consequently, in deep water the triad interactions are insignificant in comparison with the quadruplet interactions in respect of the evolution of wind waves due to energy transfer among the wave components.

However, the triad interactions may be important even in deep water for energy dissipation due to wave breaking, estimation of drag coefficients of air flow over the sea, or for navigation of ships, especially during the early stage of the development of wind waves. In deep water, waves break to form white caps if the wave steepness (or the ratio of wave height to wavelength) exceeds a certain value. Based on theoretical consideration, Michell (1893) found the limiting steepness to be 0.142 . On the other hand, using the data at Lake Ontario, Donelan (1982) showed that air flow over old wind sea has a lower drag than over young wind sea. For fixed wind speed, the drag coefficient varies due to the wave age dependence by a factor of two. For navigation of ships, the heights of waves are not as important as is their steepness. A ship can easily ride a high wave if the wave is also very long. In contrast, even a rather small wave can make trouble if it is steep. The stern gets hung on one crest, while the bow is driven under the next. As waves grow in height they also grow in length, and at a faster rate. Therefore, waves are steeper during the early stage of a blow than they are later when the sea is more fully developed. Indeed, Sverdrup and Munk (1947) showed that the wave 
steepness increases with the wave age during the early stage and then drops off as the waves grow old. The triad interactions may primarily be responsible for the steepness of deep-water wind waves.

Bispectral analysis has been utilized by a number of investigators to examine wave-wave interactions (Hasselmann et al., 1962; Masuda and Kuo, 1981b; and others). It is also very useful to estimate the magnitude of triad interactions of water waves, and several data analysis techniques have been developed for this purpose (Haubrich, 1965; Kim and Powers, 1979). In this paper, we report laboratory experiments made in a wind-wave flume to examine the triad interactions of wind waves for various water depths, wind speeds and fetch lengths. Because the length of the flume is relatively short, all the measured waves fall under deep-water conditions. At four locations along the flume, wave measurements were made for a sufficiently long time to evaluate the magnitude of triad interactions quantitatively with very small variances. The obtained wave records were then subjected to bispectral analyses to investigate the evolution of triad interactions with the wave age and so on.

\section{Triad Interactions}

Nonlinear triad interactions can occur among waves whose frequencies and wave numbers satisfy the following relationships:

$$
\begin{aligned}
& f_{1} \pm f_{2}=f_{3} \\
& \mathbf{k}_{1} \pm \mathbf{k}_{2}=\mathbf{k}_{3}
\end{aligned}
$$

where $f_{i}$ and $\mathbf{k}_{i}=$ scalar frequency and vector wave number, respectively, of the $i$ th wave component. The components 1 and 2 each obey the linear dispersion relationship:

$$
\omega^{2}=g k \tanh k d
$$

where $d=$ water depth; $g=$ gravitational acceleration; $\omega=2 \pi f ;$ and $k=|\mathbf{k}|$ = wave number magnitude. Hence, triad interactions can be interpreted as energy transfer processes among three wave components which satisfy the relationships of (1) and (2). 
Interactions where the component 3 satisfies the dispersion relationship $\left[\mathbf{k}_{3}=\mathbf{k}\left(f_{3}\right)\right]$ are termed resonant interactions (Armstrong et al., 1962). To the contrary, if it does not satisfy the dispersion relationship, the interactions are termed non-resonant or bound interactions (Hasselmann, 1962). In the resonant interactions, the amplitude of the component 3 can grow to be as large as those of the primary components 1 and 2 , whereas in the non-resonant interactions, it remains small compared with those of the primary waves. Phillips (1960) showed that for gravity surface waves the resonant interactions are only possible in very shallow water where the waves are nondispersive. In transitional or deep water, where the waves are dispersive, only non-resonant triad interactions can occur.

Triad interactions occur among a number of triads of wave components which satisfy the relationships (1) and (2). For spectra with a distinct spectral peak, however, the self interaction of the spectral peak component $\left(f_{1}=f_{2}=f_{p}\right.$ where

$f_{p}=$ frequency of the spectral peak) has been observed to be significant (e.g., Hasselmann et al., 1962; Masuda and Kuo, 1981b; Elgar and Guza, 1985; Freilich et al., 1990; Ahn, 1993; Elgar et al., 1995; Young and Eldeberky, 1998). Such a self interaction results in the generation of an harmonic of the spectral peak at $2 f_{p}$. On the other hand, in the bifrequency plane, a clear peak appears at the point $\left(f_{p}, f_{p}\right)$. In this study, we also focus our attention mainly on the self interaction of the spectral peak component.

\section{Bispectral Analysis}

As a diagnostic tool of nonlinear wave interactions, bispectral analysis is commonly used. The bispectrum is formally defined as the Fourier transform of the second-order covariance function of the time series (Hasselmann et al., 1962)

$$
B\left(f_{1}, f_{2}\right)=\int_{-\infty}^{+\infty} R\left(\tau_{1}, \tau_{2}\right) e^{-i\left[2 \pi\left(f_{1} \tau_{1}+f_{2} \tau_{2}\right)\right]} d \tau_{1} d \tau_{2}
$$

with 


$$
R\left(\tau_{1}, \tau_{2}\right)=E\left[\eta(t) \eta\left(t+\tau_{1}\right) \eta\left(t+\tau_{2}\right)\right]
$$

where $E[\cdot]$ denotes an expected value or mean; $\eta(t)=$ water surface elevation; and $\tau$ represents a time lag. For a discretely sampled time series, the bispectrum can also be expressed directly in terms of Fourier coefficients (Kim and Powers, 1979)

$$
B\left(f_{1}, f_{2}\right)=E\left[X\left(f_{1}\right) X\left(f_{2}\right) X^{*}\left(f_{1}+f_{2}\right)\right]
$$

where $X(f)$ denotes the complex Fourier coefficient and the asterisk represents the complex conjugate.

The bispectrum can be recast into two useful quantities, namely, its normalized magnitude, the bicoherence (Kim and Powers, 1979), and the biphase, which are defined respectively as

$$
\begin{aligned}
& b^{2}\left(f_{1}, f_{2}\right)=\frac{\left|B\left(f_{1}, f_{2}\right)\right|^{2}}{E\left[\left|X\left(f_{1}\right) X\left(f_{2}\right)\right|^{2}\right] E\left[\left|X\left(f_{1}+f_{2}\right)\right|^{2}\right]} \\
& \beta\left(f_{1}, f_{2}\right)=\tan ^{-1}\left\{\frac{\operatorname{Im}\left[B\left(f_{1}, f_{2}\right)\right]}{\operatorname{Re}\left[B\left(f_{1}, f_{2}\right)\right]}\right\}
\end{aligned}
$$

where the symbols $\operatorname{Re}$ and Im represent the real part and imaginary part, respectively, of a complex value. The bicoherence can be used as a measure of phase coherence between spectral components. The normalized bicoherence given by (7) varies in the range $0 \leq b^{2} \leq 1$. As a limiting case, for example, a value of $b^{2}=1$ indicates exact coherence, while $b^{2}=0$ indicates no coherence. The bicoherence given by (7) is more convenient than other normalized bispectra because it varies between 0 and 1 . If a different method of normalization is used as in Haubrich (1965) for example, the bicoherence can be larger than unity.

On the other hand, the nature of phase coherence can be investigated by the biphase. According to Masuda and Kuo (1981a), a value of $\beta=0$ is associated with the horizontally symmetric Stokes-type wave with peaked crests and flat troughs, while as $\beta$ approaches $-\pi / 2$ the waves become increasingly pitched forward. The nature of phase coherence can also be represented by such 
parameters as skewness $S_{k}$ and asymmetry $A_{s}$ :

$$
\begin{aligned}
& S_{k}=\frac{\iint \operatorname{Re}\left[B\left(f_{1}, f_{2}\right)\right] d f_{1} d f_{2}}{\left(\overline{\eta^{2}}\right)^{3 / 2}} \\
& A_{s}=\frac{\iint \operatorname{Im}\left[B\left(f_{1}, f_{2}\right)\right] d f_{1} d f_{2}}{\left(\overline{\eta^{2}}\right)^{3 / 2}}
\end{aligned}
$$

where the overbar indicates the mean with respect to time. Positive values of skewness and negligible asymmetry correspond to Stokes-type waves, while negative values of asymmetry indicate pitched-forward waves.

\section{Experiment}

\subsection{Experimental apparatus}

Experiments were carried out in the wind-wave flume at the Hydrodynamics Laboratory of the Japan Port and Harbour Research Institute. Diagrams of the flume are shown in Figs. 1 and 2. The uniform test section is $1.5 \mathrm{~m}$ wide, $1.3 \mathrm{~m}$ high and $28.5 \mathrm{~m}$ long. The side walls consist of glass plates and the top of the flume is covered with wood plates. On the windward (right hand) side of the test section over the waterway is a wind blower, which generates wind by an axial fan driven by a $50 \mathrm{~kW}$ variable-speed motor. The wind then passes through guide vanes, a fine mesh screen and honeycombs so that the wind velocity at the inlet section (see Fig. 1) is quite uniform. At the inlet, a horizontal guide plate is provided, which can be adjusted vertically so as to be located at the water surface. In this wind-wave flume, currents can also be generated by pumping water through the pipe as shown in Figs. 1 and 2. In fact, experiments were carried out with and without currents. In this paper, however, only the no-current cases are investigated.

Wind speed and water surface displacement were measured with an anemometer and resistance-type wave gauges, respectively. The data acquisition software used in the experiment directly stored the data in data files in the personal computer while displaying the data on the monitor screen in real-time during the measurement. 


\subsection{Experimental procedure}

First, wind was generated over the water and wind speeds were measured at nine elevations at an increment of $5 \mathrm{~cm}$ (i.e., at $5,10, \ldots, 45 \mathrm{~cm}$ ) above the still water level at station $\mathrm{C}$ in Fig. 2, approximately the center of the flume. When the wave height was so large that the water elevation reached the anemometer at $5 \mathrm{~cm}$, the measurement at $5 \mathrm{~cm}$ was omitted. At each elevation, measurement was made for $60 \mathrm{~s}$ at a sampling rate of $20 \mathrm{~Hz}$ to obtain the time-averaged wind speed. The vertical wind profile, $v(y)$, was determined using the equation as follows:

$$
v(y)=v_{0.5}+\frac{v_{*}}{K} \ln \left(\frac{y}{h}\right)
$$

in which $v_{0.5}=$ wind speed at $0.5 \mathrm{~m}$ above the still water level; $v_{*}=$ wind shear velocity; $y=$ vertical distance measured upward from the still water level; and $h$ was taken to be $0.5 \mathrm{~m}$ so that $v$ becomes $v_{0.5}$ at $y=0.5 \mathrm{~m}$. The quantities of $v_{0.5}$ and $v_{*}$ were calculated from the linear regression analysis of the measured wind speeds on $\ln (y / h) / K$. The thus calculated $v_{0.5}$ and $v_{*}$ are given in Table 1. An example of the measured and curve-fitted wind profiles is shown in Fig. 3. The wind profiles are not used in this study except to calculate $v_{0.5}$, which is used to calculate the wave age. The choice of the elevation of $0.5 \mathrm{~m}$ and the corresponding wind speed $v_{0.5}$ is arbitrary. But, as shown in Fig. 3, at this elevation the wind speed does not change much. Therefore, $v_{0.5}$ may represent the speed of free wind outside the boundary layer.

Second, with the wind blowing, wave measurements were made at the four locations, A, B, D and E, as shown in Fig. 2. A sufficient waiting time was allowed to elapse after the initiation of wind generation prior to data acquisition. Therefore, all the measured waves are fetch limited. A total of 36,000 data was collected at the sampling rate of $50 \mathrm{~Hz}$ for each of the four wave gauges.

The above procedure was repeated for different conditions of water depth and wind speed. Experiments were made for three different water depths; 50, 40 and $30 \mathrm{~cm}$. For the water depth of $50 \mathrm{~cm}$, eight different wind speeds were tested. For the water depths of 40 and $30 \mathrm{~cm}$, three and two different wind speeds were tested, respectively. The test conditions and the calculated parameters are given in Table 1.

\subsection{Data analysis}


The bispectra of the measured wave data were computed using the MATLAB HOSA (Higher Order Spectral Analysis) toolbox. Since the bispectral estimation has some statistical uncertainty, numerical tests were performed to confirm its validity for the real problems. The numerical test results showed that it can be used to quantitatively estimate the relative phase coherence due to wave interactions [see Oh (2000) for details of the numerical tests].

In the bispectral analysis of the wave data, of 36000 data in each test record, the first 35840 data were processed in 279 segments of 256 data per segment. These segments overlap by 50 percent for smoother and statistically more significant spectral estimates. The bispectrum was determined by ensemble averaging the Fourier coefficient product of each segment as in (6). No tapering, windowing or block averaging was applied since these processes will increase bias and reduce the ability to detect phase coherence (Nikias and Raghuveer, 1987). The frequency resolution is $\Delta f=0.1953 \mathrm{~Hz}$ and the total number of degrees of freedom is 372 for the resulting bispectra. As an example, the surface plot and contours of the bicoherence of a wave record are shown in Figs. 4 and 5, respectively. In both figures, only the values above 95 percent significant level for zero bicoherence are shown in the bifrequency octant. In this case, according to Elgar and Guza (1988), the bicoherence smaller than 0.02 is regarded to be insignificant for 372 degrees of freedom.

\section{Results}

The computed bicoherence values are quite large as shown in Figs. 4 and 5, indicating that there exist significant triad interactions between wave components. The largest bicoherence value of 0.54 is observed at $\left(f_{p}, f_{p}\right)$ and significant levels of bicoherence continue along the line of $f_{2}=f_{p}$ and $f_{1} \geq f_{p}$. This suggests that in addition to the self interaction of the spectral peak, triad interactions between the peak frequency and higher frequencies are also present. Recognizable bicoherence values along the line of $f_{2}=2 f_{p}$ are also observed but they are not so prominent as those along the peak frequency. The presence of the self interaction of the spectral peak component is also confirmed by the 
corresponding power spectrum shown in Fig. 6, where a notable secondary peak appears at the frequency of $2 f_{p}$. All other wave records yield similar results as those presented in Figs. 4 to 6.

In the analysis of surface gravity waves, the surface tension at the water surface is usually ignored because it is relatively small compared with the gravity force. However, as the scale of waves becomes small, the importance of surface tension in the wave dynamics gradually increases. In small-scale laboratory experiments, it is inevitable to observe capillary waves at locations of short fetch length, especially when the wind is relatively mild. Since capillary waves behave quite differently from gravity waves, it is necessary to discard the wave records which exhibit the characteristics of capillary waves.

To evaluate the relative importance of gravity and surface tension, the following dispersion relationship in deep water is considered (Dean and Dalrymple, 1991):

$$
C=\sqrt{\frac{g+\frac{T k^{2}}{\rho}}{k}}
$$

where $C=$ wave celerity; $\rho=$ specific density of sea water; $T=$ surface tension $(=0.074 \mathrm{~N} / \mathrm{m}$ for water in normal temperature). For pure gravity waves, the effect of surface tension disappears so that (12) is reduced to

$$
C=\sqrt{\frac{g}{k}}
$$

Hence, the contribution of surface tension to $C$ can be evaluated by the difference between (12) and (13).

There is no commonly used criterion for discriminating capillary waves and gravity waves. Moreover the effect of surface tension varies with frequency; more effects on high frequency waves. In this study, therefore, if the difference between (12) and (13) is less than 3 percent at $2 f_{p}$, the waves are considered to be gravity waves. When $f_{p}=2.35 \mathrm{~Hz}, C$ for $2 f_{p}$ is calculated to be 0.342 and 0.332 
$\mathrm{m} / \mathrm{s}$, respectively, by (12) and (13). The difference between them is about 3 percent. Therefore, the criterion for gravity waves was taken as $f_{p} \leq 2.35 \mathrm{~Hz}$. All the data at station A are capillary waves according to this criterion, so they are not included in Table 1.

All the wave data in Table 1 satisfy the deep-water condition in terms of $k_{p} d$ (i.e., $k_{p} d \geq \pi$ ). However, the low-frequency component waves may not belong to deep-water waves especially for small values of $k_{p} d$. To impose a severer restriction for deep-water condition, included in the analysis were only the wave data which satisfy the deep-water condition $(k d \geq \pi)$ at the frequency with spectral density of five percent of the peak spectral density on the lower-frequency side of the spectrum. The wave data which satisfy both this severer deep-water condition and the gravity wave condition $\left(f_{p} \leq 2.35 \mathrm{~Hz}\right)$ are marked in the last column of Table 1. Only these data are included in the following analysis.

As mentioned in the introduction, the triad interactions of deep-water wind waves may have a close relationship with the wave steepness. In Fig. 7 are shown the values of $b^{2}\left(f_{p}, f_{p}\right)$ as a function of the wave steepness $k_{p} a$, where $a=H_{s} / 2$ with $H_{s}=$ significant wave height calculated by spectral analysis. The values of $b^{2}\left(f_{p}, f_{p}\right)$ are nearly in direct proportion to $k_{p} a$, indicating that the large wave steepness results from strong triad interactions.

Sverdrup and Munk (1947) [see also Kinsman (1965), p. 308] showed theoretically that the wave steepness increases with the wave age during the early stage of generation of wind waves and then drops off as the waves grow old. They also confirmed this by gathering up available field data and plotting them. They showed that the wave steepness becomes the maximum at the wave age of about 0.4. On the other hand, by adding other field data to the Sverdrup and Munk's data, Roll (1949) [see also Wiegel (1964), p. 227] showed that the wave age for the maximum wave steepness is about 0.1 . The values of $k_{p} a$ for the present experimental data are shown as a function of the wave age $C_{p} / v_{0.5}$ in Fig. 8, 
where $C_{p}=$ phase speed of the spectral peak component. The figure shows the similar trend as those of Sverdrup and Munk (1947) and Roll (1949), being closer to the Roll's result.

Since $b^{2}\left(f_{p}, f_{p}\right)$ is almost linearly proportional to $k_{p} a$ as shown in Fig. 7, it is expected that the relationship between $b^{2}\left(f_{p}, f_{p}\right)$ and $C_{p} / v_{0.5}$ is similar to that between $k_{p} a$ and $C_{p} / v_{0.5}$. This can be confirmed in Fig. 9, which shows the values of $b^{2}\left(f_{p}, f_{p}\right)$ as a function of the wave age $C_{p} / v_{0.5}$. The magnitude of triad interactions increases with the wave age during the early stage of wave generation and then drops off as the waves grow old. The reason for this phenomenon is not quite clear. One possible explanation could be made by the difference of the time scales of triad and quadruplet interactions. According to Phillips (1960), the triad interactions transfer energy to the secondary components like a pulse, while the quadruplet interactions transfer energy to the tertiary components in a much longer time scale. The quadruplet interactions act to redistribute energy when a high spectral peak develops. It seems that a relatively large secondary spectral peak is developed by triad interactions during the early stage of wave generation until their effects are overwhelmed by the energy redistribution mechanism due to quadruplet interactions.

The nature of the phase coherence among spectral components can be represented by the biphase $\beta$, skewness $S_{k}$, and asymmetry $A_{s}$. As shown in Table 1, the absolute values of $\beta$ are much smaller than $\pi / 2$ and the magnitudes of $A_{s}$ are much smaller than those of $S_{k}$, indicating that the waves are not particularly pitched forward. In Fig. 10 is shown a part of the wave profile measured at station B for the case of $d=50 \mathrm{~cm}$ and $v_{0.5}=11.77 \mathrm{~m} / \mathrm{s}$, for which the magnitudes of both $\beta\left(f_{p}, f_{p}\right)$ and $A_{s}$ are the largest. It shows peaked crests and flat troughs but no particularly pitched-forward feature.

In Fig. 11 are shown the values of $S_{k}$ as a function of the wave steepness $k_{p} a$. As expected, they show an almost directly proportional relationship. As the waves become steep, they have more peaked crests and flatter troughs so that the 
skewness increases. Since the skewness $S_{k}$ is almost linearly proportional to the wave steepness $k_{p} a$, it is anticipated that the relationship between $S_{k}$ and $C_{p} / v_{0.5}$ is similar to that between $k_{p} a$ and $C_{p} / v_{0.5}$. The former relationship is shown in Fig. 12. The skewness increases with the wave age during the early stage of wave generation but soon drops off as the waves grow older.

The values of the bicoherence $b^{2}\left(f_{p}, f_{p}\right)$ in this experiment, ranging from 0.18 to 0.54 , are quite large compared with those of finite depth wind waves (between 0.014 and 0.166) of Young and Eldeberky (1998). One reason, as previously stated, may be that the waves are so young that the quadruplet interactions do not become fully effective yet. Another reason may be the more peaked spectral shape and smaller directional spreading in the laboratory flume compared with those in the field. Masuda and Kuo (1981b) theoretically showed that the magnitude of bispectrum increases as the frequency spectrum becomes more peaked and the directional spreading becomes smaller. As shown in Table 1, in our laboratory experiment, the values of the peak enhancement factor, $\gamma$, are very large compared with those in the field. The typical value of $\gamma$ is 3.3 for wind waves in developing stage (e.g., the JONSWAP spectrum) and is 1.0 for fully developed wind waves. The directional spreading was not measured in the present experiment. However, it is expected that the directional spreading must be very small in the one-dimensional laboratory flume compared with that in the field.

\section{Conclusions}

The triad interactions of deep-water wind waves were investigated using the bispectral analysis of the waves measured in a laboratory wind-wave flume. To reduce the variance of the bispectral estimates of the wave records, the duration of the wave measurement was kept long enough to obtain sufficient degrees of freedom. Included in the analysis were only the records of sufficiently developed waves, which show the characteristics of gravity waves.

It was shown that there is an almost directly proportional relationship between any two of $b^{2}\left(f_{p}, f_{p}\right), k_{p} a$, and $S_{k}$, and that all these increase with the wave age during the early stage of wave generation and then drop off as the waves grow 
old. The latter relationship has been observed by Sverdrup and Munk (1947) and Roll (1948) between the wave steepness and wave age. From the results of the present study, therefore, we could conclude that the steep waves with peaked crests and flatter troughs during the early stage of the generation of wind waves are resulted from the triad interactions.

The large values of $b^{2}\left(f_{p}, f_{p}\right)$ during the early stage of wave generation may be related to the peaked spectral form and the narrow directional spreading compared with those in the field. On the other hand, the decrease of $b^{2}\left(f_{p}, f_{p}\right)$ with the wave age may be related to the effect of quadruplet interactions, which act to redistribute energy among the wave components. To examine the relative importance between triad and quadruplet interactions, other diagnostic tools such as trispectral analysis should be utilized (Elgar et al., 1995).

The triad interactions have been known to be important only for shoaling waves or finite depth wind waves. In this study, we have shown that they are also significant for deep-water wind waves especially during the early stage of wave development. When the waves are more fully developed, energy may still be transferred from the spectral peak to the second harmonic $\left(2 f_{p}\right)$ by triad interactions, which may then be redistributed to the neighboring frequencies by quadruplet interactions. These processes may act to elevate the spectral tail along the high frequencies. The details of how bound energy near $2 f_{p}$ would be influenced by quadruplet interactions are yet to be investigated.

\section{Acknowledgements}

Funding for this research from the Japan Ministry of Transport and the Korea Ocean Research and Development Institute (under Contract No. 99-027 of the Research Institute of Engineering Science, Seoul National University) is gratefully acknowledged. The first and second writers were also supported by the Japan Science and Technology Agency and the Japan Port and Harbour Research Institute (PHRI), respectively, for their visit to the PHRI. The writers would like to thank Messrs. Yasuyuki Nakagawa, Masayoshi Suzuki, Masaharu Kudo, Takayuki 
Nomura, and Yuji Sato for their help during the experiment. 


\section{References}

Ahn, K. (1993). Nonlinear analysis of waves in finite water depth, $\mathrm{PhD}$ thesis, Univ. of Florida, Gainesville, 61p.

Armstrong, J. A., Bloembergen, N., Ducing, J. and Pershan, P. S. (1962). Interactions between light waves in a nonlinear dielectric, Phys. Rev., 127: 1918-1939.

Dean, R. G. and Dalrymple, R. A. (1991). Water Wave Mechanics for Engineers and Scientists, World Scientific, Singapore, 353p.

Donelan, M. A. (1982). The dependence of the aerodynamics drag coefficient on wave parameters, Proc. 1st Int. Conf. on Meteorological and Air/Sea Interaction of Coastal Zone, Amer. Meteor. Soc., Boston, pp. 381-387.

Elgar, S. and Guza, R. T. (1985). Observations of bispectra of shoaling surface gravity waves, J. Fluid Mech., 161: 425-448.

Elgar, S. and Guza, R. T. (1988). Statistics of bicoherence, IEEE Trans. Acoust. Speech Signal Process., 36(10): 1667-1668.

Elgar, S., Herbers, T. H. C., Chandran, V. and Guza, R. T. (1995). Higher-order spectral analysis of nonlinear ocean surface gravity waves, J. Geophys. Res., 100: $4977-4983$.

Freilich, M. H., Guza, R. T. and Elgar, S. (1990). Observations of nonlinear effects in directional spectra of shoaling surface gravity waves, J. Geophys. Res., 95: 9645-9656.

Hasselmann, K. (1962). On the non-linear energy transfer in a gravity-wave spectrum: 1. General theory, J. Fluid Mech., 12: 481-500.

Hasselmann, K., Munk, W. and MacDonald, G. (1962). Bispectra of ocean waves, Proc., Symp. on Time Series Analysis, John Wiley, New York, pp. 125-139.

Haubrich, R. A. (1965). Earth noise, 5 to 500 millicycles per second, J. Geophys. Res., 70: 1415-1427.

Kim, Y. C. and Powers, E. J. (1979). Digital bispectral analysis and its applications to nonlinear wave interactions, IEEE Trans. Plasma Sci., PS-7(2): 120-131.

Kinsman, B. (1965). Wind Waves, Their Generation and Propagation on the Ocean Surface, Prentice-Hall, New Jersey, 676p.

Masuda, A. and Kuo, Y.-Y. (1981a). A note on the imaginary part of bispectra, Deep Sea Res., 28A(3): 213-222.

Masuda, A. and Kuo, Y.-Y. (1981b). Bispectra for the surface displacement of random gravity waves in deep water, Deep Sea Res., 28A(3): 223-237.

Michell, J. H. (1893). On the highest waves in water, Philosophical Magazine, 5th 
Series, 36: 430-437.

Nikias, C. L. and Raghuveer, M. R. (1987). Bispectrum estimation: A digital signal processing framework, Proc. IEEE, 75(7): 869-891.

Oh, S. H. (2000). Influence of currents on equilibrium range spectra and triad interactions of finite depth wind waves, Master thesis, Seoul Nat. Univ., Seoul, $80 \mathrm{p}$.

Phillips, O. M. (1960). On the dynamics of unsteady gravity waves of finite amplitude. Part 1. The elementary interactions, J. Fluid Mech., 9: 193-217.

Roll, H. U. (1949). Über die ausbreitung der meereswellen unter der wirkung des windes (auf grund von messungen im wattenmeer), Deut. Hydrograph. Z., 2(6): 268-280.

Sverdrup, H. U. and Munk, W. H. (1947). Wind, sea and swell: theory of relations for forecasting, U.S. Navy Hydrographic Office Pub. No. 601, 44p.

Wiegel, R. L. (1964). Oceanographical Engineering, Prentice-Hall, New Jersey, $532 \mathrm{p}$.

Young, I. R. and Eldeberky, Y. (1998). Observations of triad coupling of finite depth wind waves, Coast. Engrg., 33: 137-154. 


\section{Captions of Figures}

1. Plan of wind-wave flume

2. Illustration of experimental setup

3. Measured and curve-fitted wind profile $\left(d=50 \mathrm{~cm}, v_{0.5}=8.73 \mathrm{~m} / \mathrm{s}\right)$

4. Surface plot of bicoherence at station $\mathrm{E}\left(d=40 \mathrm{~cm}, \quad v_{0.5}=12.11 \mathrm{~m} / \mathrm{s}\right)$

5. Contour plot of bicoherence at station $\mathrm{E}\left(d=40 \mathrm{~cm}, v_{0.5}=12.11 \mathrm{~m} / \mathrm{s}\right)$

6. Power spectrum at station E $\left(d=40 \mathrm{~cm}, \quad v_{0.5}=12.11 \mathrm{~m} / \mathrm{s}\right)$

7. Values of $b^{2}\left(f_{p}, f_{p}\right)$ as function of wave steepness $k_{p} a$

8. Values of $k_{p} a$ as function of wave age $C_{p} / v_{0.5}$

9. Values of $b^{2}\left(f_{p}, f_{p}\right)$ as function of wave age $C_{p} / v_{0.5}$

10. Measured wave profile at station B $\left(d=50 \mathrm{~cm}, v_{0.5}=11.77 \mathrm{~m} / \mathrm{s}\right)$

11. Values of $S_{k}$ as function of wave steepness $k_{p} a$

12. Values of $S_{k}$ as function of wave age $C_{p} / v_{0.5}$ 


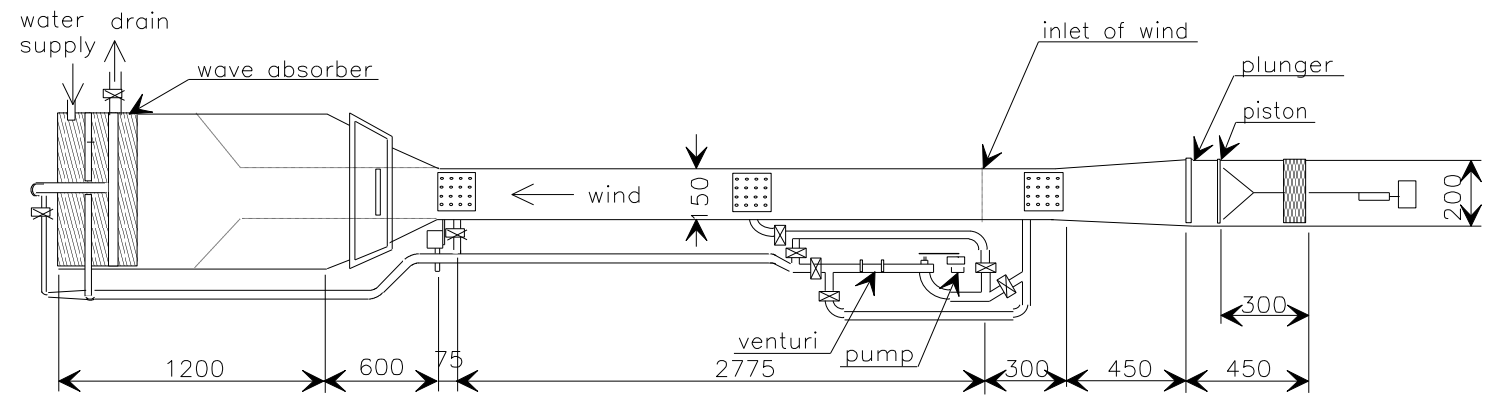

Fig.1 Plan of wind-wave flume.

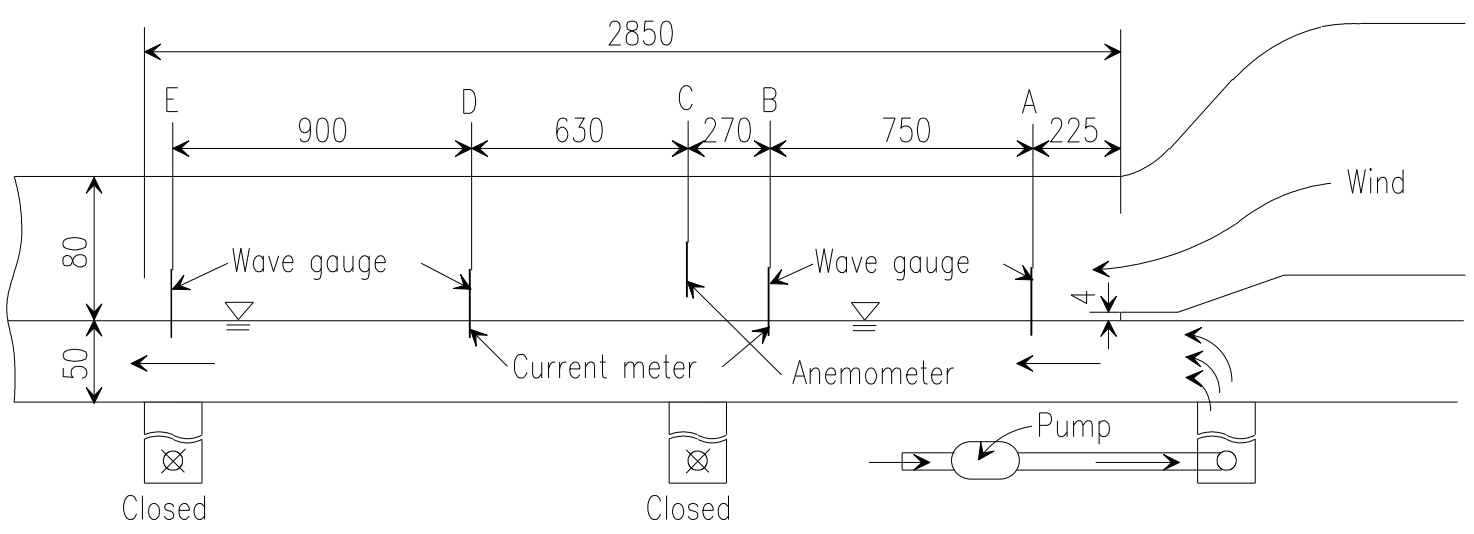

Fig.2 Illustration of experimental setup. 
Fig. 3

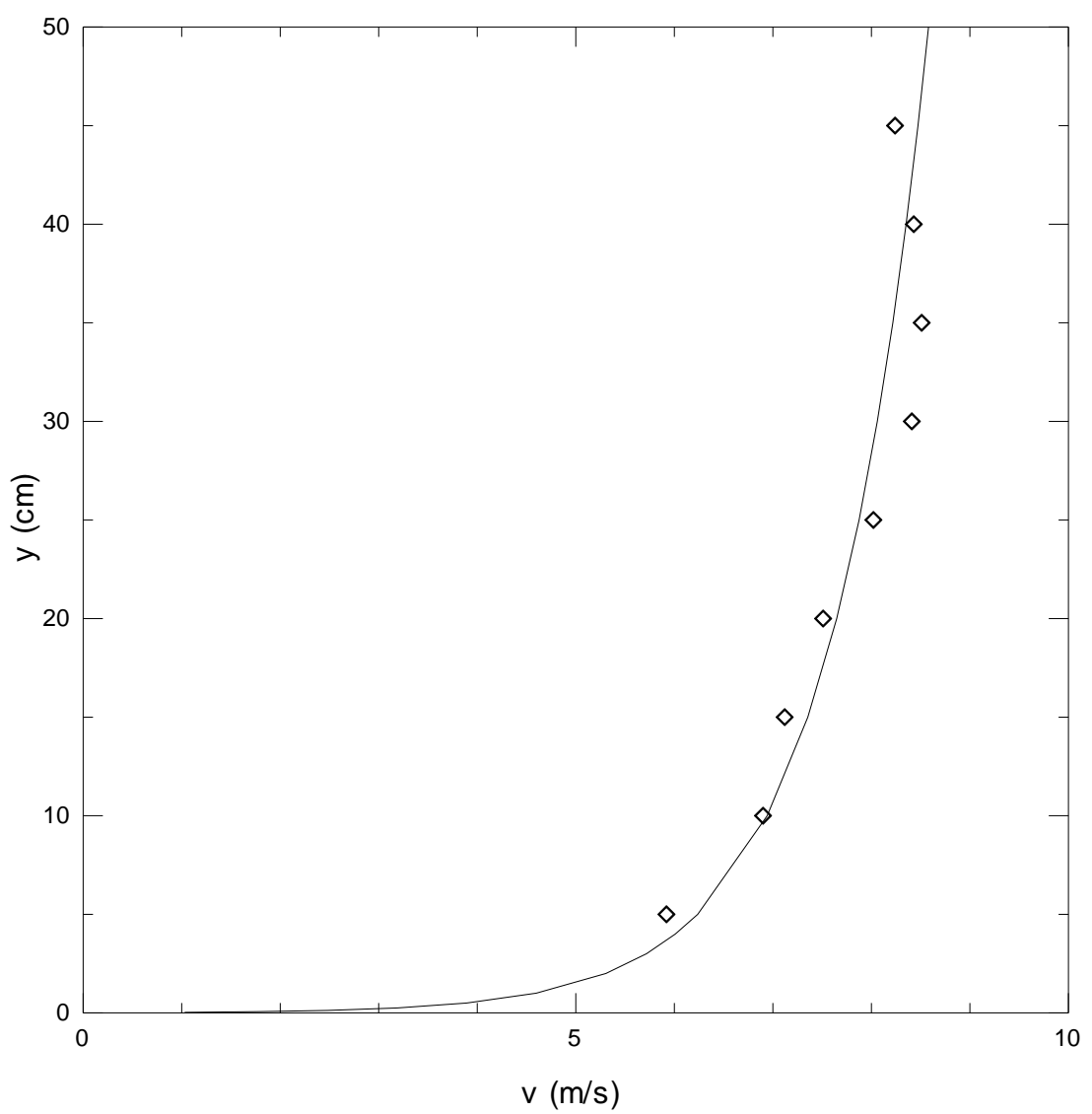


Fig. 4

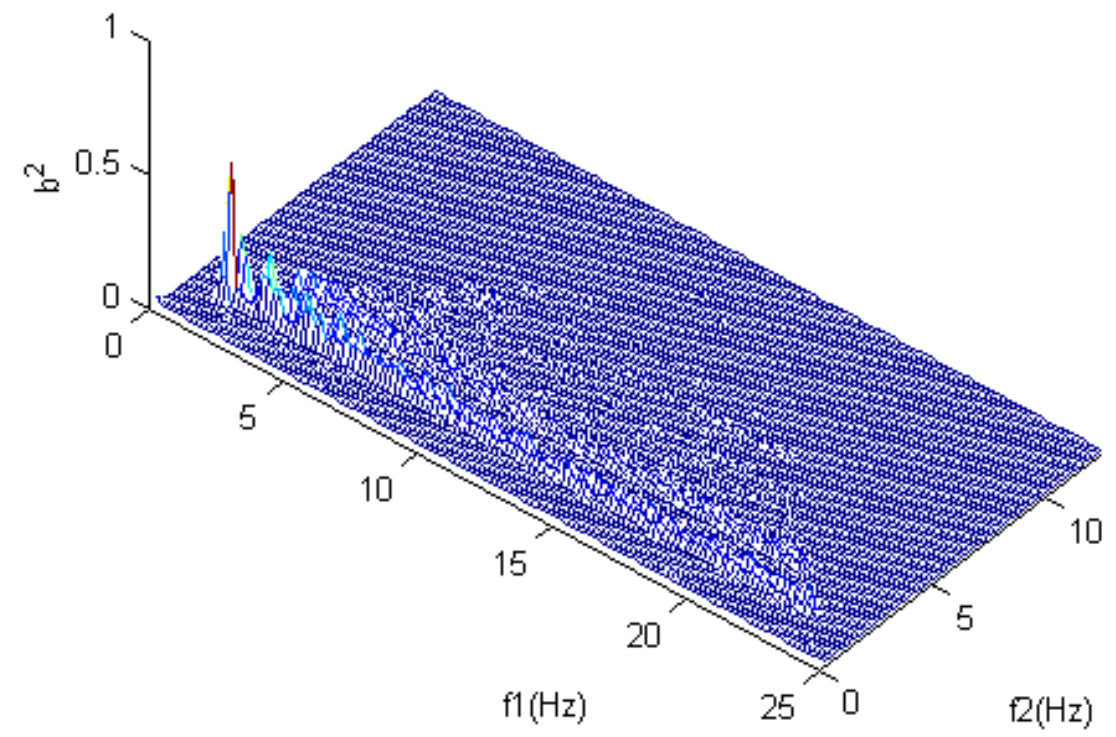


Fig. 5

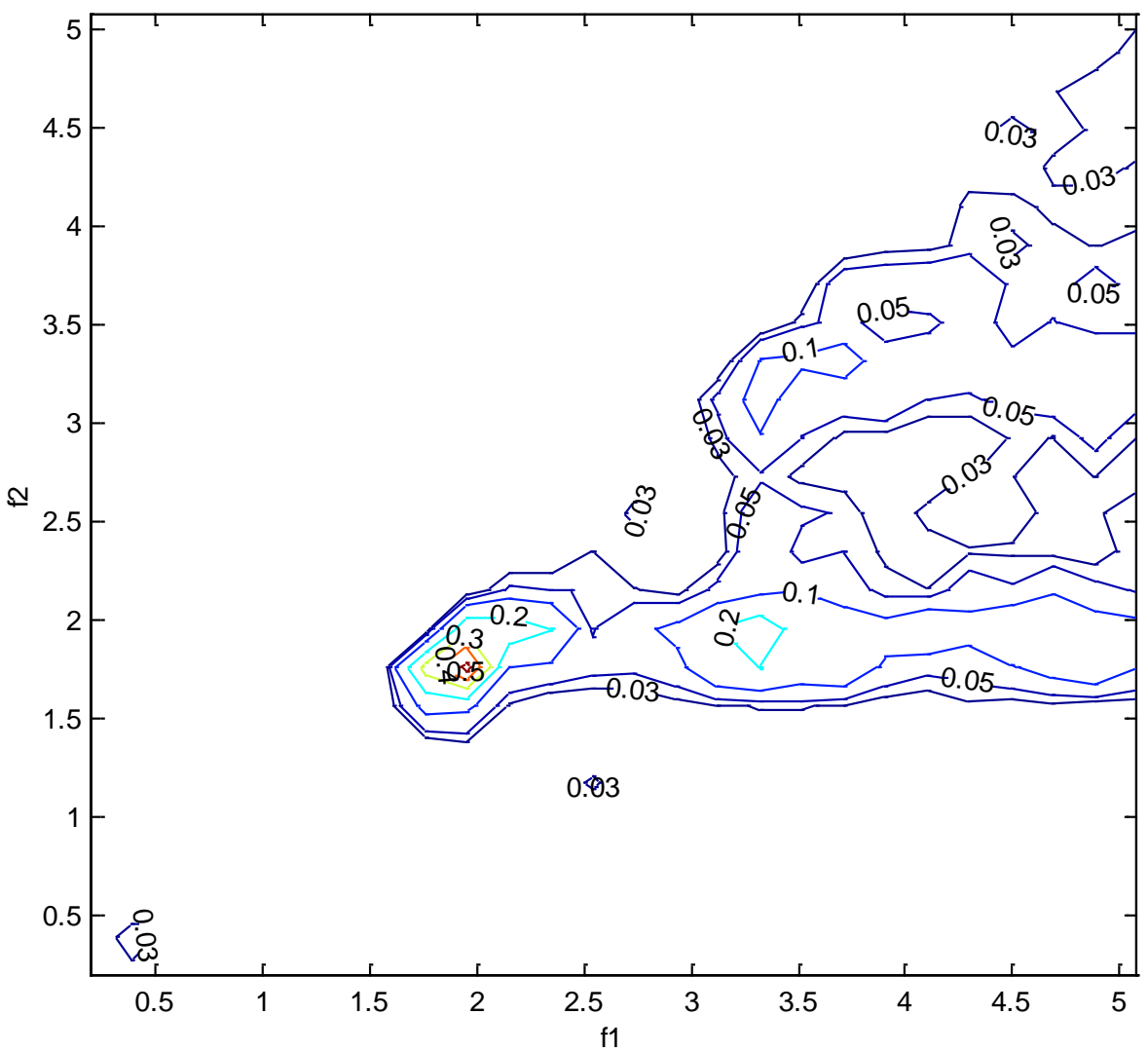


Fig. 6

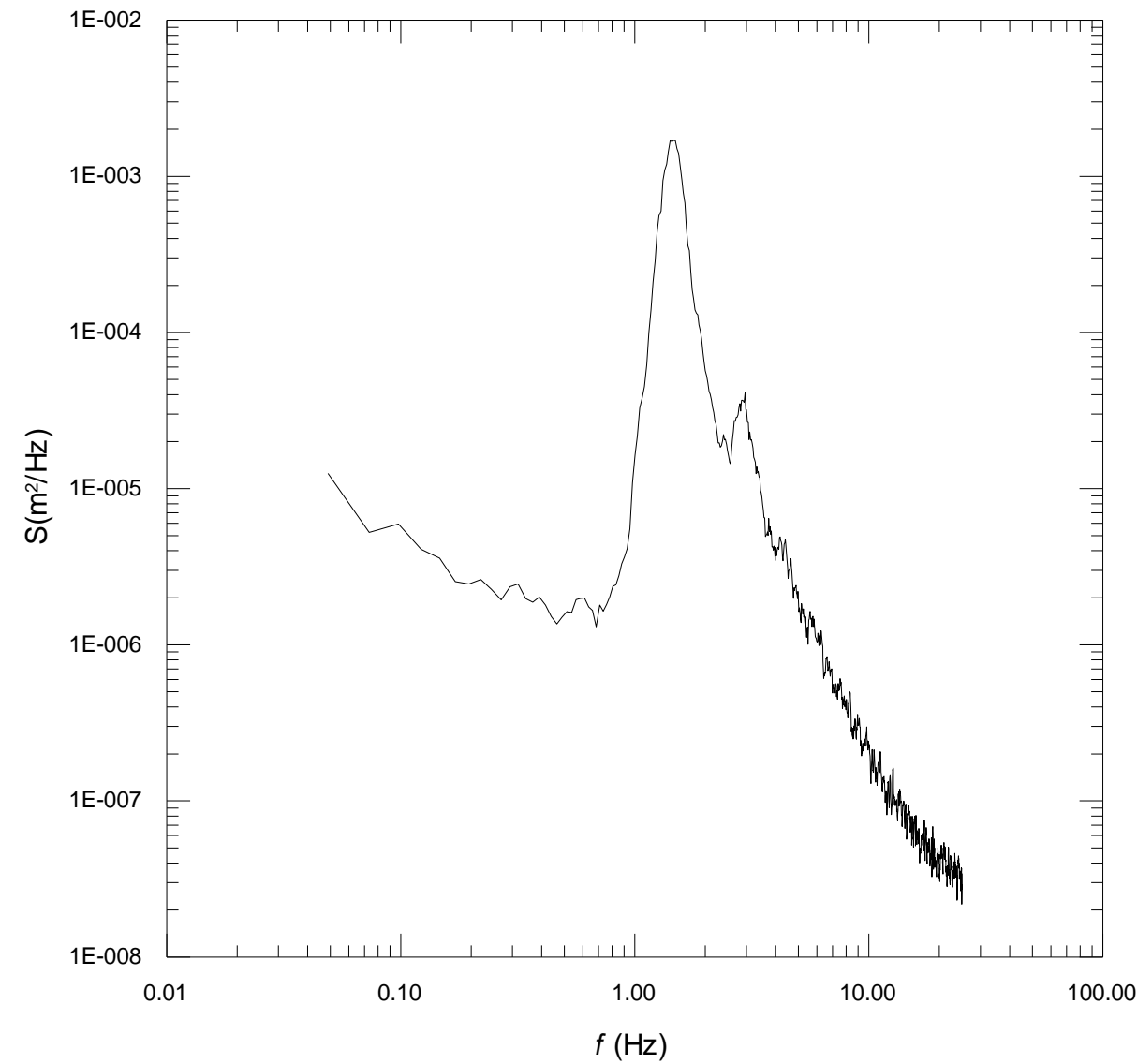


Fig. 7

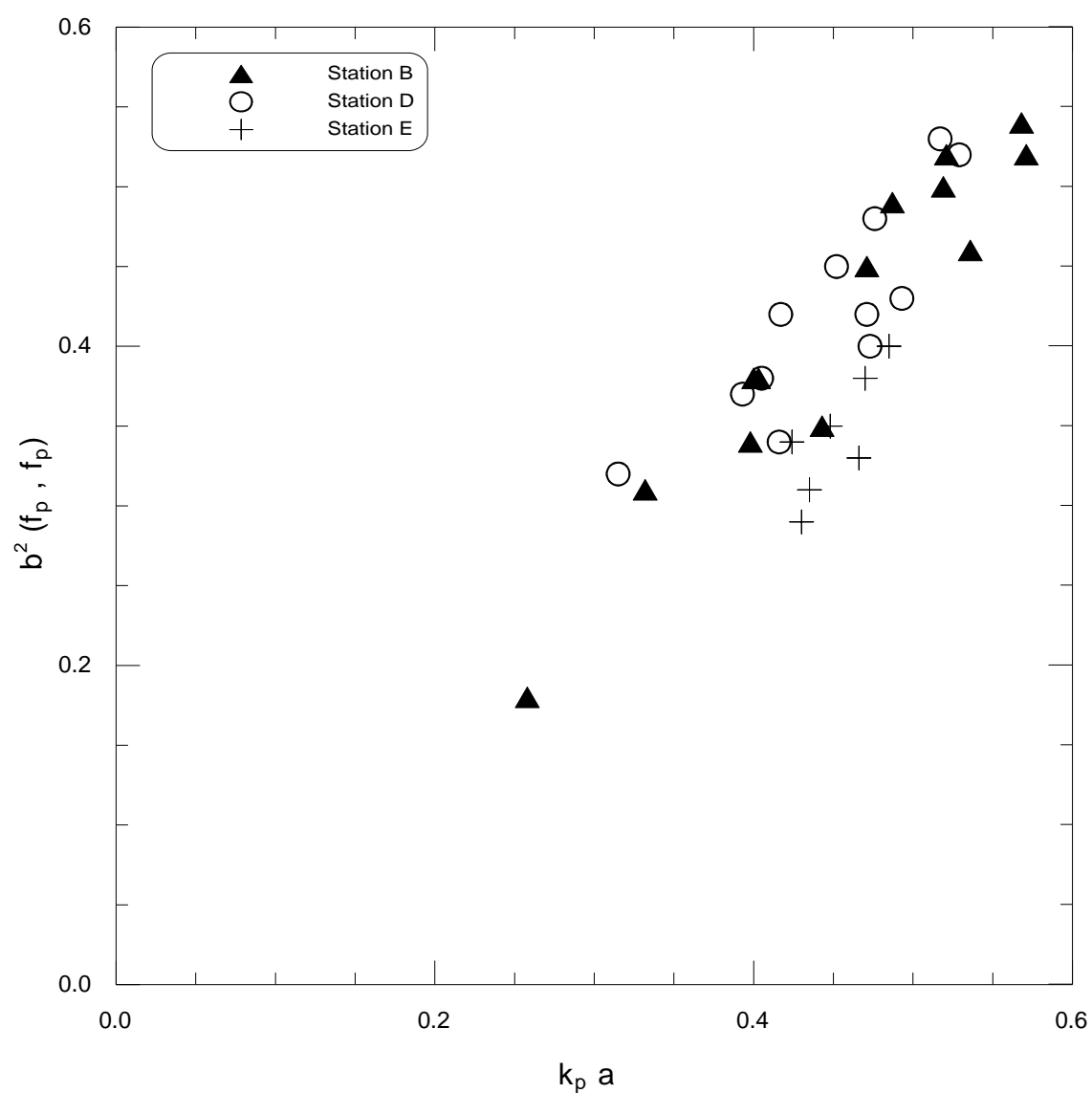


Fig. 8

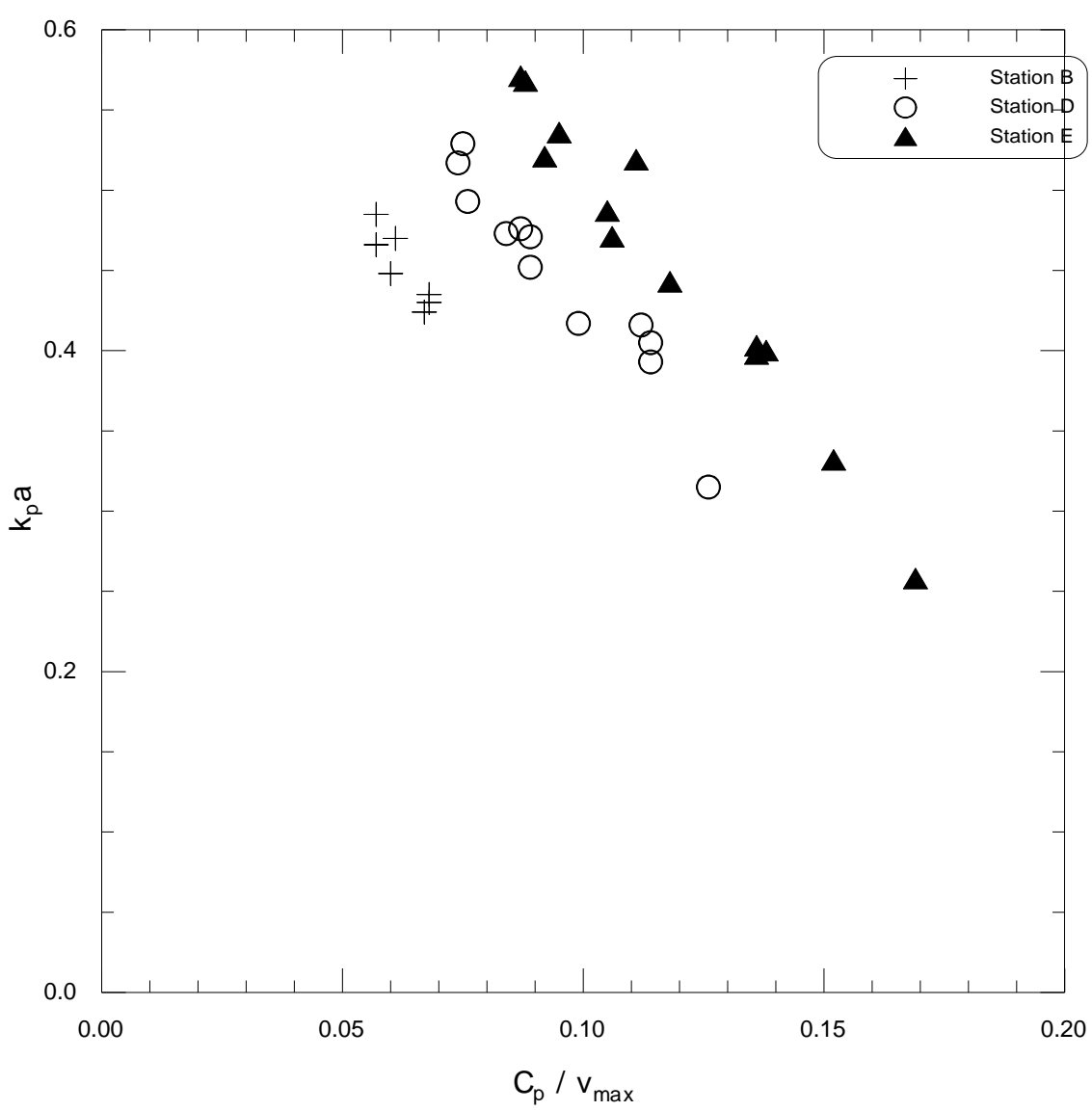


Fig. 9

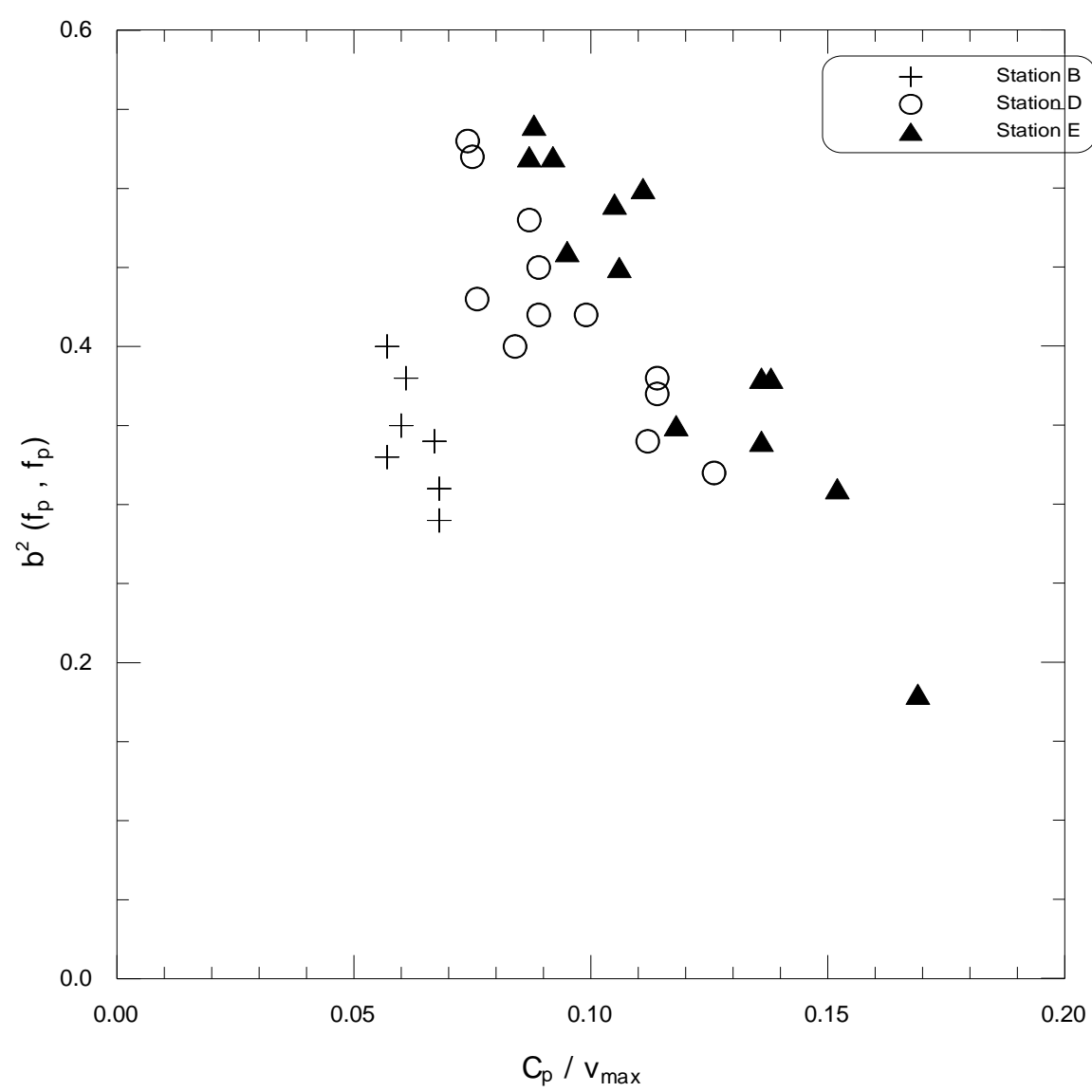


Fig. 10

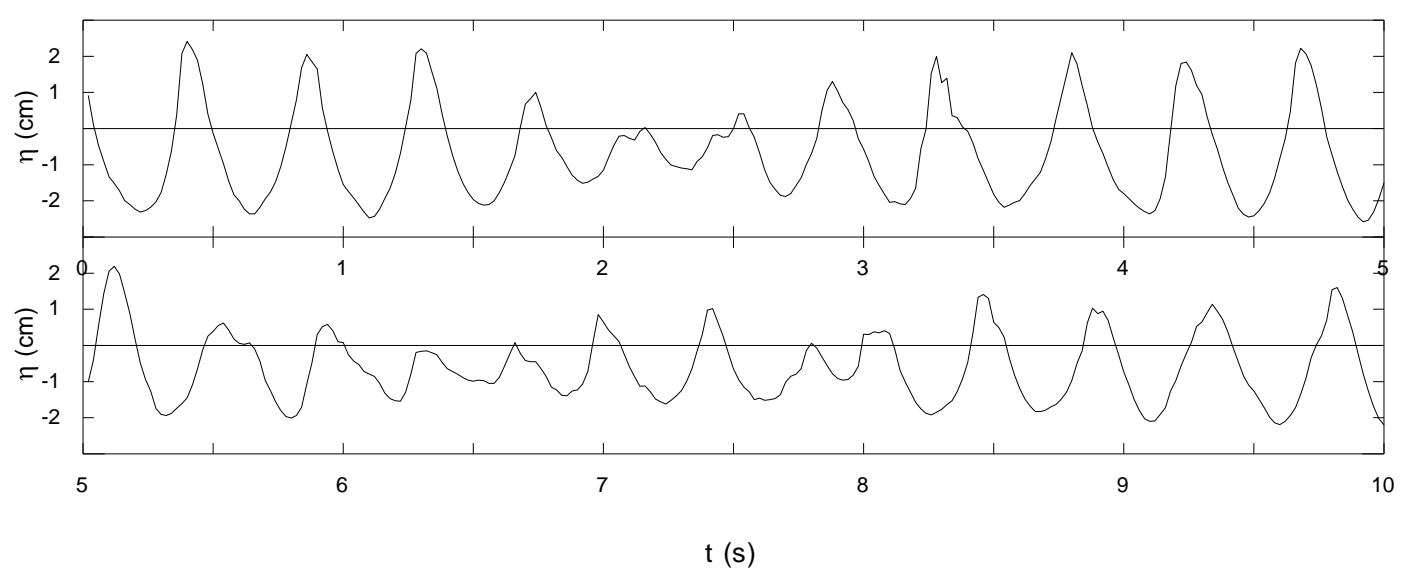


Fig. 11

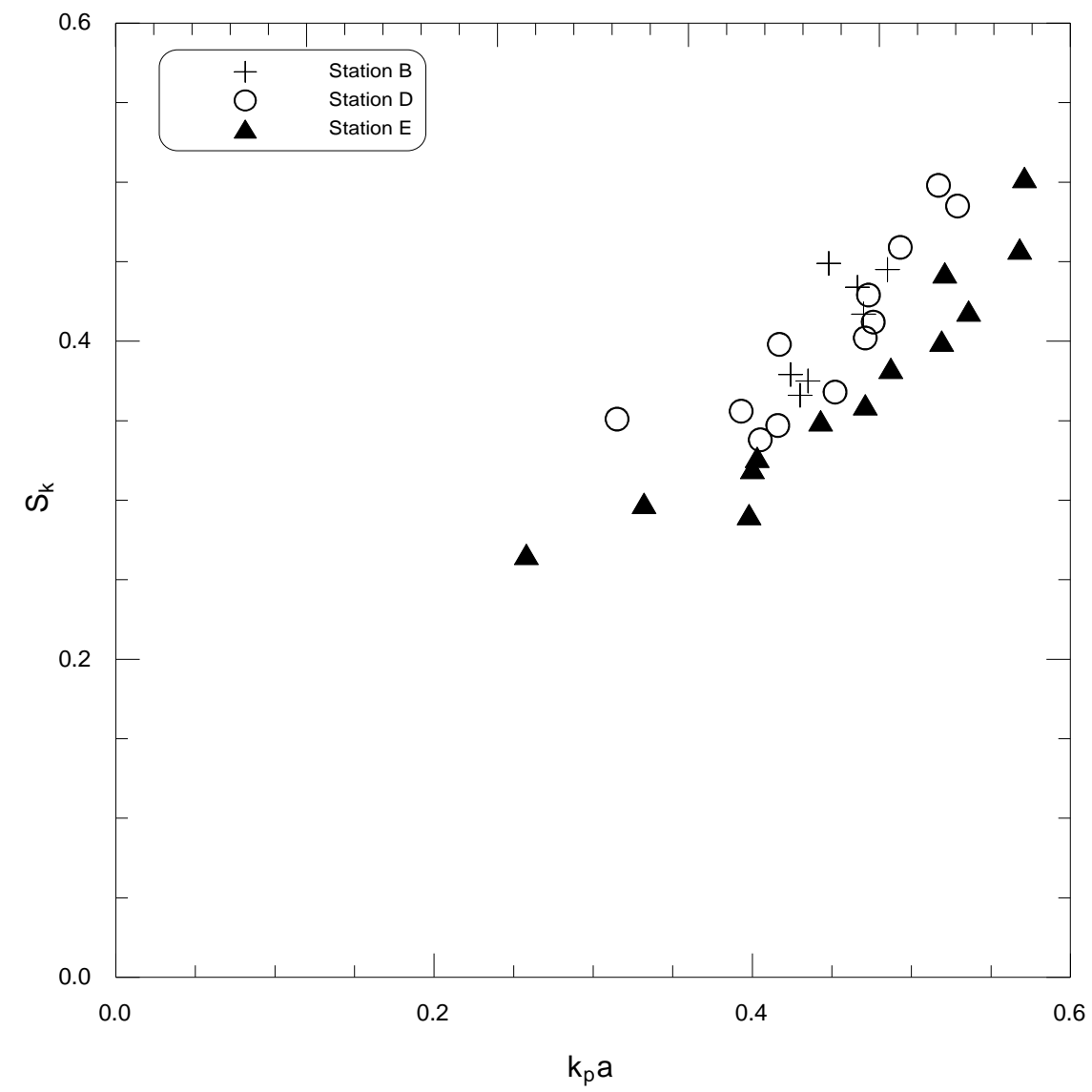


Fig. 12

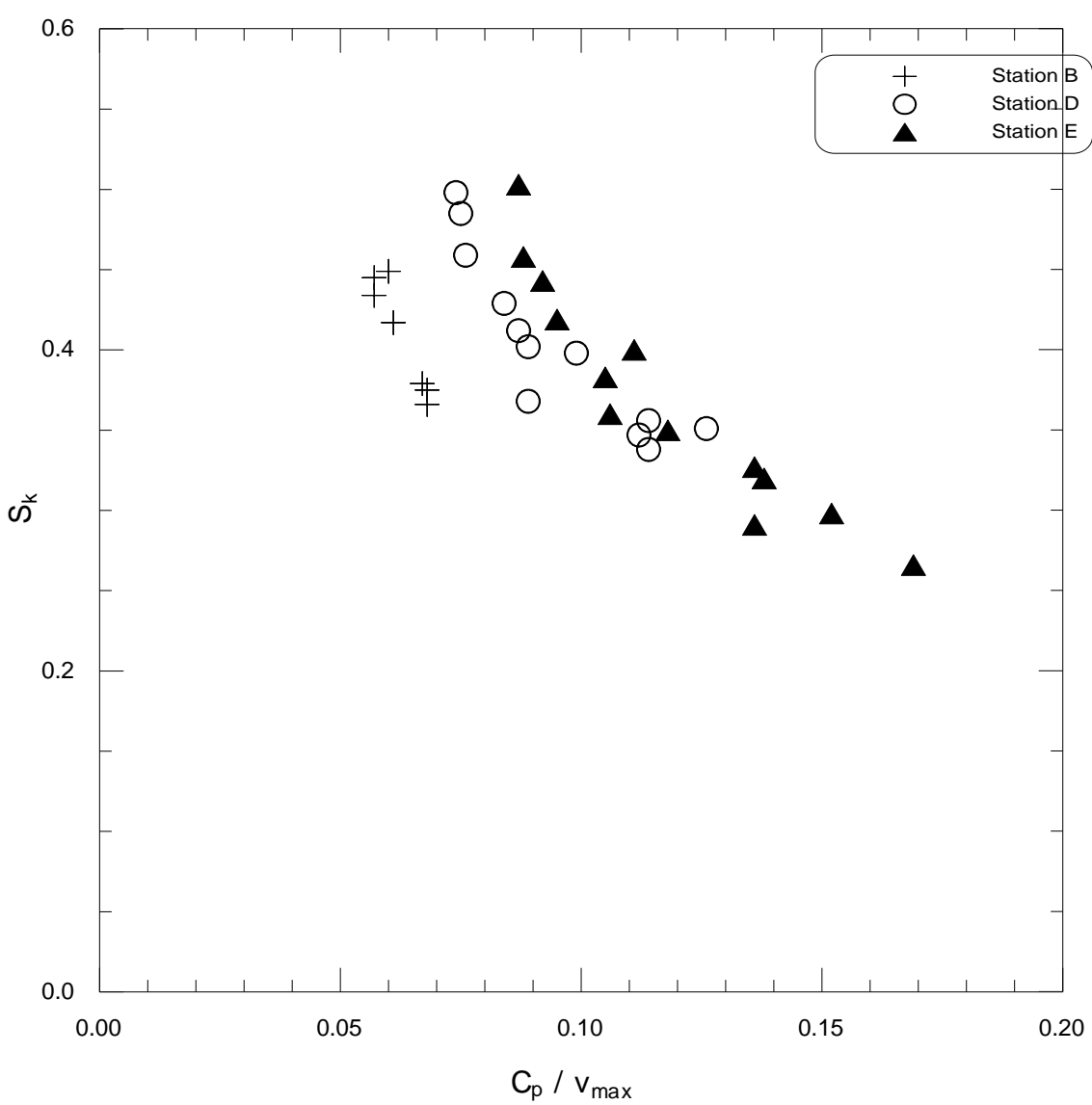

